\title{
Hemophagocytic Lymphohistiocytosis; A Rare Complication Of Dengue Haemorrhagic Fever
}

\section{Chamila Pradeep}

Teaching Hospital Peradeniya

Parackrama Karunathilake ( $\sim$ parackramawk@gmail.com )

University of Peradeniya Faculty of Medicine https://orcid.org/0000-0002-7029-7781

Shamali Abeygunawardena

University of Peradeniya Faculty of Medicine

Udaya Ralapanawa

University of Peradeniya Faculty of Medicine

\section{Thilak Jayalath}

University of Peradeniya Faculty of Medicine

\section{Research Article}

Keywords: Hemophagocytic Lymphohistiocytosis, Dengue, Cytopenia, Dexamethasone

Posted Date: September 8th, 2021

DOI: https://doi.org/10.21203/rs.3.rs-734782/v1

License: (c) (1) This work is licensed under a Creative Commons Attribution 4.0 International License. Read Full License 


\section{Abstract \\ Background}

Haemophagocytic Lymphohistiocytosis (HLH) is an uncommon systemic inflammatory clinical syndrome associated with numerous conditions and a rare complication of dengue associated with significant mortality and morbidity even with appropriate treatment. The outcome is further poor if the diagnosis is delayed or left untreated. Therefore a high degree of clinical suspicion is paramount in the diagnosis of $\mathrm{HLH}$, especially in an atypical presentation of dengue infection.

\section{Case Presentation}

A 17-year-old boy with a non-significant past medical history admitted with a four-day history of fever, headache, nausea, vomiting, and loose stool. On admission, he was hemodynamically stable and managed as serologically confirmed dengue fever. On the 5th day of fever, he entered the critical phase, which was uncomplicated except for high fever spicks. Even after the critical phase was over, he had ongoing high fever spicks, hepatosplenomegaly with persistent thrombocytopenia, neutropenia and anaemia. Serum ferritin level was $>3000 \mathrm{ng} / \mathrm{ml}$, while the triglyceride level was $314 \mathrm{mg} / \mathrm{dl}$. Bone marrow biopsy revealed an increased haemophagocytic activity. Secondary HLH was diagnosed based on criteria used in the HLH-2004 trial and successfully managed with intravenous dexamethasone $10 \mathrm{mg} /$ body surface area/day for the first two weeks followed by tapering of course over eight weeks.

\section{Conclusion}

Though this is a rare complication, the clinician must suspect HLH in expanded dengue syndrome complicated with ongoing fever, splenomegaly with cytopenias, and necessary investigations to establish a firm diagnosis. Early treatments will result in promising outcomes while preventing complications and reducing mortality.

\section{Background}

Haemophagocytic Lymphohistiocytosis $(\mathrm{HLH})$, also known as the hemophagocytic syndrome, is an uncommon systemic inflammatory clinical syndrome associated with numerous conditions, such as neoplastic, infectious, autoimmune, or hereditary diseases [1]. HLH may occur as inherited (primary) or secondary to any severe infection, malignancy or rheumatologic condition [2]. Dengue is a rising pathogen in tropical countries, giving rise to rare serious complications that clinicians must know [3]. HLH is sporadically seen in clinical practice and a rare complication of dengue that presents persistent fever, pancytopenia, hepatosplenomegaly, and increased serum ferritin. Owing to the fact the overlap in clinical features, it has its diagnostic difficulty when present in a dengue patient and requires bone marrow examination [4]. Although secondary $\mathrm{HLH}$ is a rare complication, it associated with significant mortality 
and morbidity even with appropriate treatment, and the outcome is further poor if the diagnosis is delayed or left untreated [5]. Therefore a high degree of clinical suspicion is paramount in the diagnosis of $\mathrm{HLH}$, especially in the case of an atypical presentation of an underlying medical condition [2]. Here we present a case of HLH in an adolescent, which occurred secondary to dengue haemorrhagic fever, which was successfully treated and recovered.

\section{Case Presentation}

A 17-year-old boy was admitted to the medical ward with a four-day history of high-grade intermittent fever associated with chills associated with nausea, few episodes of vomiting and watery diarrhoea for the same duration. He also complained of severe frontal headache with retro-orbital pain, arthralgia, myalgia, and anorexia on direct inquiry. However, abdominal cramps or right hypochondriacal pain was not there on admission. He denied any respiratory symptoms or urinary symptoms, and his urine output was normal. Apart from headache, there were no other neurological symptoms, including photophobia or phonophobia. He was a well and active school student until he developed this acute febrile illness, and he did not have any significant past medical history

He was mildly dehydrated with a capillary refilling time of fewer than 2 seconds, and he was not pale or icteric. There was no lymphadenopathy. His pulse rate was 100 beats per minute with a $110 / 70 \mathrm{mmHg}$ blood pressure without any postural drop. Respiratory and abdominal examination was unremarkable, and there was no hepatosplenomegaly.

In the initial full blood count, he had a white cell count of $2.7 \times 10^{9} / \mathrm{L}$, with neutrophils $1.9 \times 10^{9} / \mathrm{L}$ and lymphocyte $0.6 \times 10^{9} / \mathrm{L}$, and a red blood cell count of $5.04 \times 10^{9} / \mu \mathrm{L}$. Haemoglobin level was $14.7 \mathrm{~g} / \mathrm{dL}$, with a haematocrit of $41.5 \%$ and the platelet count was $125 \times 10^{3} / \mu \mathrm{L}$. The C-reactive protein (CRP) level was $30.9 \mathrm{mg} / \mathrm{L}$, aspartate aminotransferase (AST) $1716 \mathrm{U} / \mathrm{L}$ and alanine aminotransferase (ALT) 822 $\mathrm{U} / \mathrm{L}$. The dengue NS1 antigen was positive.

After the initial workup, he was diagnosed with dengue fever, and dengue febrile phase monitoring was started. On the following day, he entered into the critical phase of dengue fever, which was uncomplicated: routine fluid management and monitoring were done. However, even after the critical phase of dengue was over, he had continuous high-grade fever with persistent thrombocytopenia and gradual haemoglobin drop. His red blood cell count was $3.21 \times 10^{6} / \mu \mathrm{L}$, and the platelet count was $27 \times 10^{3} / \mu \mathrm{L}$. He had also developed mild to moderate hepatosplenomegaly. The blood picture revealed thrombocytopenia with giant platelets and features suggestive of HLH. His serum ferritin level was > $3000 \mathrm{ng} / \mathrm{mL}(10-300 \mathrm{ng} / \mathrm{mL})$, triglycerides $314 \mathrm{mg} / \mathrm{dL}$ and the bone marrow biopsy revealed features of significant haemophagocytic activity. Based on these investigation findings, he was diagnosed to have $\mathrm{HLH}$.

Then he was started with intravenous dexamethasone $10 \mathrm{mg}$ per body surface area $(17 \mathrm{mg})$ for the first two weeks and then converted to oral dexamethasone, which was gradually tailed off over eight weeks. 
There was a good response clinically as well as biochemically. The fever settled within 24 hours after starting intravenous dexamethasone with a doubling of white cell and platelet count. He was discharged after a two-week hospital stay.

\section{Discussion}

$\mathrm{HLH}$ is a rare, potentially fatal hyperinflammatory syndrome, causing severe hypercytokinemia with excessive activation of lymphocytes and macrophages [2]. It is also known as a hemophagocytic syndrome associated with numerous conditions, such as neoplastic, infectious, autoimmune, or hereditary diseases [1]. The disease is seen in all ages and has no predilection for race or sex [6]. There are two main types; primary or familial HLH associated with genetic predisposition and secondary or sporadic HLH associated with other medical conditions, including infective, rheumatoid, and malignant conditions [7].

The designations primary and secondary HLH have become less relevant, and instead, genetic and acquired HLH is more appropriate [1]. Acquired (secondary) HLH can occur in all age groups, although there are no published data on incidence or age distribution. Conditions associated with acquired HLH are predominantly infections, autoimmune diseases and, especially in adults, malignant diseases [8]. The infections causing HLH include viral infections like herpesviruses, human immunodeficiency virus (HIV), adenovirus, dengue and hantavirus, bacterial causes like Staphylococcus aureus, Campylobacter spp, and Mycoplasma spp, fungal infections like Candida spp, Cryptococcus spp, Pneumocystis spp, and Histoplasma spp, and parasitic infections like Plasmodium falciparum, Plasmodium vivax, Toxoplasma spp, and Leishmania spp [1]. HLH is an uncommon manifestation in dengue, and the diagnosis of HLH is difficult when present concurrent with dengue fever $[4,9]$.

Dengue fever is caused by the Dengue virus, which belongs to the family Flaviviridae, genus Flavivirus, and is transmitted to humans by Aedes mosquitoes, mainly Aedes aegypti [10]. The clinical spectrum of dengue viral infection includes undifferentiated fever, dengue fever, dengue haemorrhagic fever (DHF) and expanded dengue syndrome or isolated organopathy [11]. Those infected with the dengue virus, especially for the first time, having a simple fever indistinguishable from other viral infections is called undifferentiated fever $[11,12]$. Dengue fever is an acute febrile illness with severe headache, myalgia, arthralgia and rashes associated with leucopenia and thrombocytopenia $[10,11,13]$. DHF is characterized by the acute onset of high fever associated with signs and symptoms similar to DF in the early febrile phase, with plasma leakage giving rise to a tendency to develop hypovolemic shock (dengue shock syndrome) [10-12]. Expanded dengue syndromes are used to designate cases that do not fall into either dengue shock syndrome or dengue hemorrhagic fever, associated with atypical and unusual manifestations [14]. Our patient demonstrated classic symptoms of dengue such as fever, severe frontal headache with retro-orbital pain, arthralgia, and myalgia, although also had nausea and watery diarrhoea. After the clinical suspicion, confirmation of dengue infection can be done by detecting the virus, viral nucleic acid, antigens or antibodies, or combining these techniques [15]. The virus can be detected in circulation within the first $4-5$ days by checking for NS1 antigen $[11,15]$. IgM antibodies are detectable in 
$50 \%$ of patients by days $3-5$ after the onset of illness, increasing to $80 \%$ by day five and $99 \%$ by day ten, where its peak about two weeks after the onset of symptoms and then decline generally to undetectable levels over 2-3 months. Anti-dengue serum IgG is generally detectable at low titres at the end of the first week of illness, increasing slowly after that, with serum IgG still detectable after several months, and probably even for life [15]. In our patient, the NS1 antigen positivity confirmed the diagnosis of dengue infection, facilitating further management.

Persistent fever following dengue infection indicates the vital differential diagnosis of sepsis and expanded dengue syndrome, including $\mathrm{HLH}$ [16-18]. HLH is an unusual haematological manifestation of expanded dengue syndrome, among other manifestations like disseminated intravascular coagulopathy (DIC) and cytopenias [14]. There are several reported cases of HLH as a secondary manifestation of dengue infection both in the paediatric and adult population. Most of these cases have occurred in patients without any other comorbidities; however, one case has reported co-infection with Plasmodium vivax, and another case has occurred in a young child with beta-thalassemia major [3, 9, 19-25].

The pathogenesis of HLH was at first thought to be a result of an inability to clear infections in immunodeficient patients [26]. However, HLH in immunocompetent patients worked to disprove such a theory later, and the identification of cytotoxic pathway mutations as the primary cause of genetic HLH has elucidated to some extent the mechanism of this disease [1]. All forms of HLH are thought to be due to impairment in the function of cytotoxic T lymphocytes and natural killer (NK) cells, associated with a potentially fatal cytokine storm and hyperferritinemia [1,27]. However, the exact mechanism is less precise for nongenetic forms of $\mathrm{HLH}$ [8]. The inability to clear the antigenic stimulus and thus turn off the inflammatory response ultimately leads to the hypercytokinemia characteristic of $\mathrm{HLH}$ [28]. If antigen removal is inefficient, as in individuals with HLH-causing mutations in the cytotoxic pathway, the inflammatory stimulus will not be terminated, resulting in a final common pathway of HLH with uncontrolled hypercytokinemia, sustained macrophage activation and tissue infiltration [28-30].

The clinical features of HLH appear to be due to CD8 + T-cell expansion, activation and infiltration of visceral organs associated with macrophage activation and the release of multiple cytokines and chemokines [2]. The initial symptoms of HLH are nonspecific and may overlap with other inflammatory or hematopoietic diseases, and the diagnosis of $\mathrm{HLH}$ is based on the diagnostic criteria as revised for $\mathrm{HLH}-$ 2004 [7, 31]. According to HLH-2004, there are two main criteria; Criterion 1 and Criterion 2. The diagnosis of HLH can be established if Criterion 1 or 2 is fulfilled. Criterion 1 includes a molecular diagnosis consistent with $\mathrm{HLH}$. The Criterion 2 includes fulfilling five out of the eight criteria, namely fever, splenomegaly, cytopenias (affecting 2 of 3 lineages in the peripheral blood, haemoglobin $<9 \mathrm{~g} / \mathrm{dL}$, platelets $<100 \times 10^{9} / \mathrm{L}$, and neutrophils $<1.0 \times 10^{9} / \mathrm{L}$ ), hypertriglyceridemia or hypofibrinogenemia (fasting triglycerides $\geq 3.0 \mathrm{mmol} / \mathrm{L}$ (i.e., $\geq 265 \mathrm{mg} / \mathrm{dL}$ ), fibrinogen $\leq 1.5 \mathrm{~g} / \mathrm{L}$ ). Hemophagocytosis in bone marrow or spleen or lymph nodes, no evidence of malignancy, low or no NK cell activity (according to local laboratory reference), ferritin $\geq 500 \mathrm{mg} / \mathrm{L}$ and SCD25 (i.e., soluble IL-2 receptor) $\geq 2400 \mathrm{U} / \mathrm{mL}$ are also included in Criterion 2 [7]. Our patient developed continuous high-grade fever and thrombocytopenia, anaemia and mild to moderate hepatosplenomegaly where the blood picture revealed thrombocytopenia 
with giant platelets and features suggestive of HLH. His serum ferritin and triglyceride levels were high, fulfilling the criteria for the diagnosis of HLH. However, the bone marrow biopsy was also done, although it was unnecessary since the diagnosis could already be made with available information. However, since in dengue infection, pancytopenia can happen as a complication of the disease itself bone marrow examination can be justified to confirm the diagnosis and to start appropriate therapy [3]. Bone marrow examination in our patient revealed significant haemophagocytic activity which confirmed the diagnosis.

The management principles of HLH include suppression of hyper inflammation, elimination of activated immune cells, elimination of trigger, supportive therapy (neutropenia, coagulopathy) and replacement of defective immune system $[1,5,25,27]$. Immediate suppression of severe hyper inflammation should be done to prevent life-threatening outcomes of $\operatorname{HLH}[1-3,8]$. The treatment protocol includes induction, salvage, and continuation therapies [2, 25]. Suppression of hyper inflammation and the elimination of activated immune cells can be achieved with corticosteroids, intravenous immunoglobulins, cyclosporin $A$, anti-cytokine agents like etoposide, and monoclonal antibodies alemtuzumab, and rituximab $[5,7,27]$. Corticosteroids are the first choice to suppress hypercytokinemia, primarily dexamethasone; since dexamethasone crosses the blood-brain barrier better than prednisolone, therefore it can suppress inflammation in the CNS more effectively [8]. The 2004 treatment protocol formulated at the second international meeting of the Histiocyte Society recommends an 8-week induction therapy with corticosteroids, etoposide, and cyclosporine A as the backbone of HLH treatment [1, 29]. The trigger can be eliminated by anti-infectious therapy and replacing a defective immune system with a haemopoietic stem cell transplant $[5,27]$. Therefore, the HLH-2004 guideline recommends chemo-immunotherapy with etoposide, dexamethasone, cyclosporine A upfront, and intrathecal therapy with methotrexate in selected patients corticosteroids. Subsequent hematopoietic stem cell transplantation (HSCT) is recommended for patients with familial disease or molecular diagnosis and severe and persistent, or reactivated, disease [7]. In patients with milder forms of HLH, corticosteroids with or without immunoglobulins may be sufficient to control hyper inflammation; however, initially, mild cases can deteriorate rapidly within a short time [8].

Regarding the treatment of dengue associated $\mathrm{HLH}$, some recovered spontaneously with supportive treatment only. However, in most cases, the pulse dosage of methylprednisolone or dexamethasone has been used to suppress the hyperinflammatory state. The treatment of dengue induced HLH by intravenous immunoglobulin $\mathrm{G}$ seems to be associated with a favourable outcome [23]. HLH in dengue patients respond well to the conventional treatment of $\mathrm{HLH}$ [9]. Importantly, HLH-directed treatment with dexamethasone and etoposide showed substantially reduced mortality in potentially fatal viral infections associated with HLH [32].

Primary HLH has a near $100 \%$ fatality without adequate treatment. However, in international studies (HLH-94/HLH-2004), survival increased from 0-60\% with HLH-directed treatment, including dexamethasone and the cytotoxic drug etoposide. [33] Even untreated, secondary FHL is a rapidly fatal disease. Most patients die from bacterial or fungal infections due to prolonged neutropenia, multiorgan failure, or cerebral dysfunction. [6] Therefore, prompt initiation of treatment is essential for the survival of 
the affected patient, and the clinical course of HLH may be very aggressive. Sometimes initial treatment may be necessary to prevent early fatalities, even though the diagnostic workup has not been completed $[3,29]$. After the initial diagnosis of $\mathrm{HLH}$, our patient was started with intravenous dexamethasone for the first two weeks and then converted to oral dexamethasone, which was gradually tailed off over eight weeks. The patient had an excellent response to treatment only with dexamethasone.

\section{Conclusion}

$\mathrm{HLH}$ is a rare, highly fatal unusual manifestation of dengue infection. The diagnosis of HLH should always be suspected when persistent fever follows recovery from dengue fever, particularly when persistent pancytopenia aided by other investigations such as serum triglyceride, fibrinogen, and ferritin levels. Early recognition and prompt institution of appropriate immunosuppressive therapy is the most vital factor for achieving a good prognosis. The diagnosis is usually challenging, as it can be easily mistaken with other clinical situations, such as sepsis. Bone marrow examination is essential when a dengue patient develops HLH to confirm the diagnosis since both conditions can give rise to pancytopenia. Corticosteroids are the first choice to suppress hypercytokinemia, primarily dexamethasone, since dexamethasone crosses the blood-brain barrier better than prednisolone, along with other immunosuppressants such as etoposide, and cyclosporine $A$ and haemopoietic stem cell transplant stands out as the curative treatment.

\section{Abbreviations}

HIV: Human Immunodeficiency Virus; HLH: Haemophagocytic Lymphohistiocytosis; HSCT: Haemopoietic Stem Cell Transplant; CRP: C-Reactive Protein; AST: Aspartate aminotransferase; ALT: Alanine aminotransferase; DF: Dengue Fever; DHF: Dengue Haemorrhagic Fever; DIC: Disseminated Intravascular Coagulation

\section{Declarations}

\section{Ethics approval and consent to participate}

Not applicable

\section{Consent for publication}

Informed written consent was obtained from the patient for publication of this case report.

\section{Availability of data and materials}

The authors confirm that the data supporting the findings of this study are available within the article.

\section{Competing interests}


The authors declare that they have no competing interests.

\section{Funding}

This study was self-funded by the investigators. No external organization or institution was involved in this study.

\section{Authors' Contributions}

All authors involved in the management of the patient and generating the concept. All authors made an intellectual contribution and wrote the paper. All authors read and approved the final manuscript.

\section{Acknowledgements}

We express our gratitude to the patient who kindly gave consent for this case to be presented in this paper.

\section{Authors' information}

Chamila Pradeep (MBBS, MD) is a Registrar in Medicine at Teaching Hospital Peradeniya, Sri Lanka. Parackrama Karunathilake (MBBS) is a Temporary Lecturer at, Department of Medicine, Faculty of Medicine, University of Peradeniya, Sri Lanka. Shamali Abeygunawardena [MBBS, MD, MRCP (UK), FRCP (London)] is a Senior Lecturer and Senior Consultant Physician, Department of Medicine, Faculty of Medicine, University of Peradeniya, Sri Lanka. Udaya Ralapanawa [MBBS, MD, MRCP (UK), FRCP (London), FRCP (Edinburgh)] is a Professor in Medicine and Senior Consultant Physician, Department of Medicine, Faculty of Medicine, University of Peradeniya, Sri Lanka. Thilak Jayalath [MBBS, MD, MRCP (UK), FRCP (London), FRCP (Edinburgh), FCCP (Sri Lanka)] is a Professor in Medicine and Senior Consultant Physician, Department of Medicine, Faculty of Medicine, University of Peradeniya, Sri Lanka.

\section{References}

1. Rosado FGN, Kim AS. Hemophagocytic lymphohistiocytosis. Am J Clin Pathol. 2013;139:713-27.

2. Gupta S, Weitzman S. Primary and secondary hemophagocytic lymphohistiocytosis: Clinical features, pathogenesis and therapy. Expert Rev Clin Immunol. 2010;6:137-54.

3. De Koninck AS, Dierick J, Steyaert S, et al. Hemophagocytic lymphohistiocytosis and dengue infection: Rare case report. Acta Clin Belg [Internet]. 2014 [cited 2021 Jul 4];69:210-213. Available from: https://pubmed.ncbi.nlm.nih.gov/24820925/.

4. Wiwanitkit V. Haemophagocytic lymphohistiocytosis and dengue [Internet]. Acta Clin. Belgica Int. J. Clin. Lab. Med. Taylor and Francis Ltd.; 2015 [cited 2021 Jul 4]. p. 72. Available from: https://www.tandfonline.com/doi/abs/10.1179/2295333714Y.0000000040.

5. Janka GE, Lehmberg K. Hemophagocytic lymphohistiocytosis: pathogenesis and treatment. [Internet]. Hematology Am. Soc. Hematol. Educ. Program. Hematology Am Soc Hematol Educ 
Program; 2013 [cited 2021 Jul 2]. p. 605-611. Available from:

https://pubmed.ncbi.nlm.nih.gov/24319239/.

6. Janka GE. Familial and acquired hemophagocytic lymphohistiocytosis [Internet]. Annu. Rev. Med. Annu Rev Med; 2012 [cited 2021 Jul 4]. p. 233-246. Available from: https://pubmed.ncbi.nlm.nih.gov/22248322/.

7. Henter JI, Horne AC, Aricó M, et al. HLH-2004: Diagnostic and therapeutic guidelines for hemophagocytic lymphohistiocytosis [Internet]. Pediatr. Blood Cancer. Pediatr Blood Cancer; 2007 [cited 2021 Jul 2]. p. 124-131. Available from: https://pubmed.ncbi.nlm.nih.gov/16937360/.

8. Janka G. Hemophagocytic lymphohistiocytosis: When the immune system runs amok [Internet]. Klin. Padiatr. Georg Thieme Verlag; 2009 [cited 2021 Jul 4]. p. 278-285. Available from: https://pubmed.ncbi.nlm.nih.gov/19707989/.

9. Kapdi M, Shah I. Dengue and haemophagocytic lymphohistiocytosis. Scand J Infect Dis [Internet]. 2012 [cited $2021 \mathrm{Jul}$ 4];44:708-709. Available from: https://pubmed.ncbi.nlm.nih.gov/22264033/.

10. Martina BEE, Koraka P, Osterhaus ADME. Dengue virus pathogenesis: An integrated view [Internet]. Clin. Microbiol. Rev. American Society for Microbiology (ASM); 2009 [cited 2021 Jul 5]. p. 564-581. Available from: /pmc/articles/PMC2772360/.

11. Ministry of Health Sri Lanka. Guidelines on Management of Dengue Fever \& Dengue Haemorrhagic Fever In Adults [Internet]. 2010 [cited 2021 Jul 3]. Available from: .

12. Kosakai N. Clinical diagnosis. Kango Kyoshitsu. 1968;12:26-9.

13. Ustafa M. Dengue Fever: Clinical Spectrum, And Management. IOSR J Dent Med Sci. 2017;16:53-9.

14. Umakanth M, Suganthan N. Unusual Manifestations of Dengue Fever: A Review on Expanded Dengue Syndrome. Cureus [Internet]. 2020 [cited 2021 Jul 5];12. Available from: https://www.cureus.com/articles/40499-unusual-manifestations-of-dengue-fever-a-review-onexpanded-dengue-syndrome.

15. WHO. LABORATORY DIAGNOSIS AND DIAGNOSTIC TESTS - Dengue - NCBI Bookshelf [Internet]. 2009 [cited 2021 Jul 5]. Available from: https://www.ncbi.nlm.nih.gov/books/NBK143156/.

16. Teparrukkul P, Hantrakun V, Day NPJ, et al. Management and outcomes of severe dengue patients presenting with sepsis in a tropical country. PLoS One [Internet]. 2017 [cited 2021 Jul 6];12. Available from: /pmc/articles/PMC5402971/.

17. Syue LS, Tang HJ, Hung YP, et al. Bloodstream infections in hospitalized adults with dengue fever: Clinical characteristics and recommended empirical therapy. J Microbiol Immunol Infect. 2019;52:225-32.

18. Gulati S, Maheshwari A. Atypical manifestations of dengue. Trop Med Int Heal. 2007;12:1087-95.

19. Ren D, Ong SWX, Batac JAL, et al. Haemophagocytic lymphohistiocytosis in dengue fever [Internet]. Lancet Infect. Dis. Lancet Publishing Group; 2021 [cited 2021 Jul 3]. p. 437. Available from: https://pubmed.ncbi.nlm.nih.gov/33639131/. 
20. Ray S, Kundu S, Saha M, et al. Hemophagocytic syndrome in classic dengue fever. J Glob Infect Dis [Internet]. 2011 [cited 2021 Jul 6];3:399-401. Available from: /pmc/articles/PMC3250000/.

21. Chung SM, Song JY, Kim W, et al. Dengue-associated hemophagocyticlymphohistiocytosis in an adult A case report and literature review. Med (United States) [Internet]. 2017 [cited $2021 \mathrm{Jul} 6$ ];96. Available from: https://journals.Iww.com/mdjournal/Fulltext/2017/02240/Dengue_associated.17.aspx.

22. Thadchanamoorthy V, Dayasiri K. Dengue Fever Associated Haemophagocytic Lymphohistiocytosis: A Report of Two Children. Cureus [Internet]. 2020 [cited 2021 Jul 3];12. Available from: https://pubmed.ncbi.nlm.nih.gov/33269160/.

23. Ray U, Dutta S, Mondal S, et al. Severe dengue due to secondary hemophagocytic lymphohistiocytosis: A case study. IDCases [Internet]. 2017 [cited 2021 Jul 6];8:50-53. Available from: /pmc/articles/PMC5388930/.

24. Chang CY, Rajappan M, Zaid M, et al. Dengue fever complicated by hemophagocytic lymphohistiocytosis: Report of 2 cases and bone marrow findings. Clin Case Reports [Internet]. 2020 [cited $2021 \mathrm{Jul}$ 6];8:3427-3431. Available from: https://orcid.

25. Khurram M, Faheem M, Umar M, et al. Hemophagocytic Lymphohistiocytosis Complicating Dengue and Plasmodium vivax Coinfection. Case Rep Med. 2015;2015.

26. Deane S, Selmi C, Teuber SS, et al. Macrophage activation syndrome in autoimmune disease [Internet]. Int. Arch. Allergy Immunol. Int Arch Allergy Immunol; 2010 [cited 2021 Jul 4]. p. 109-120. Available from: https://pubmed.ncbi.nlm.nih.gov/20407267/.

27. Rosée P, La, Horne AC, Hines M, et al. Recommendations for the management of hemophagocytic lymphohistiocytosis in adults. Blood [Internet]. 2019 [cited 2021 Jul 4];133:2465-2477. Available from: http://ashpublications.org/blood/article-pdf/133/23/2465/1553600/blood894618.pdf.

28. Grom AA. Natural Killer Cell Dysfunction: A Common Pathway in Systemic-Onset Juvenile Rheumatoid Arthritis, Macrophage Activation Syndrome, and Hemophagocytic Lymphohistiocytosis? [Internet]. Arthritis Rheum. Arthritis Rheum; 2004 [cited 2021 Jul 4]. p. 689-698. Available from: https://pubmed.ncbi.nlm.nih.gov/15022306/.

29. Aricò $M$, Danesino $C$, Pende $D$, et al Pathogenesis of haemophagocytic lymphohistiocytosis [Internet]. Br. J. Haematol. Br J Haematol; 2001 [cited 2021 Jul 4]. p. 761-769. Available from: https://pubmed.ncbi.nlm.nih.gov/11564062/.

30. Jordan MB, Hildeman D, Kappler J, et al. An animal model of hemophagocytic lymphohistiocytosis $(\mathrm{HLH}): \mathrm{CD} 8+\mathrm{T}$ cells and interferon gamma are essential for the disorder. Blood [Internet]. 2004 [cited 2021 Jul 4];104:735-743. Available from: https://pubmed.ncbi.nlm.nih.gov/15069016/.

31. Jordan $\mathrm{MB}$, Allen $\mathrm{CE}$, Greenberg $\mathrm{J}$, et al. Challenges in the diagnosis of hemophagocytic lymphohistiocytosis: Recommendations from the North American Consortium for Histiocytosis (NACHO) [Internet]. Pediatr. Blood Cancer. John Wiley and Sons Inc.; 2019 [cited 2021 Jul 2]. Available from: https://pubmed.ncbi.nlm.nih.gov/31339233/. 
32. Gao C, Cai Y, Zhang K, et al. Association of hypertension and antihypertensive treatment with COVID19 mortality: a retrospective observational study. Eur Heart J [Internet]. 2020 [cited $2021 \mathrm{Jul}$ 5];41:2058-2066. Available from: /pmc/articles/PMC7314067/.

33. Kan FK, Tan CC, von Bahr Greenwood T, et al. Dengue Infection Complicated by Hemophagocytic Lymphohistiocytosis: Experiences from 180 Patients with Severe Dengue. Clin Infect Dis [Internet]. 2020 [cited 2021 Jul 6];70:2247-2255. Available from: https://academic.oup.com/cid/article/70/11/2247/5514267.

\section{Supplementary Files}

This is a list of supplementary files associated with this preprint. Click to download.

- CareCheckListHLHinDengue.docx 